\title{
REMARQUES CONCERNANT LE FONCTIONNEMENT DES MANDIBULES CHEZ LES FEMELLES DE DIPTÈRES HÉMATOPHAGES NÉMATOCÈRES ET BRACHYCÈRES
}

\author{
Par P. GRENIER
}

Divers travaux de Gordon et coll. (1939, 1948, 1953) ont attiré l'attention des parasitologues sur les mécanismes qui permettent à certains Diptères hématophages (Aedes aegypti L., Cimex, Chrysops, Glossina) de percer la peau de leur hôte pour effectuer leurs repas de sang. Ces auteurs ont insisté sur l'intérêt qu'offre la connaissance précise de tels mécanismes en ce qui concerne l'ingestion et l'inoculation de germes pathogènes par l'insecte. Dans leur revue de la littérature consacrée à ces questions, les chercheurs de la Liverpool School of Tropical Medicine ont fait remarquer que les idées classiques sur le fonctionnement des pièces buccales étaient fondées sur des études anatomiques, alors que les observations directes d'insectes en train de piquer étaient très rares. L'interprétation de ces mécanismes est done très conjecturale et les observations de Gordon et ses collaborateurs ont prouvé que les idées courantes étaient erronées.

Les remarques que nous nous proposons de formuler ici ne sont pas non plus fondées sur des observations directes effectuées sur des insectes en train de se nourrir. Nous voulons simplement montrer que diverses explications sont inexactes parce que certaines particularités morphologiques ont été mal étudiées et parce que des observations déjà anciennes n'ont pas été prises en considération et sont même complètement tombées dans l'oubli.

L'idée de cette révision nous est venue en relisant les travaux des auteurs (notamment Nitzulescu, Jobling, Gibbins) qui ont étudié l'armement buccal des femelles de Nématocères hématophages (Phlebotomina, Ceratopogonidre, Simuliidæx). Nous avons ainsi constaté comment une interprétation très probablement fausse, celle des mandibules disposées en « ciseaux » et sectionnant la peau en pivotant sur un axe central, a presque réussi à s'imposer malgré les restrictions formulées par l'éminent morphologiste Snodgrass. 
Il nous a semblé intéressant de procéder à une révision critique de ces travaux, en y ajoutant les quelques observations personnelles que nous avons été amené à faire chez les Nématocères et les Brachycères. Une telle revue permettra peut-être de rendre à certains travaux. la place qu'ils auraient dû. avoir dans la littérature scientifique consacrée aux Diptères ; elle montrera, nous l'espérons, l'intérêt qu'offre toujours la lecture attentive de publications déjà anciennés.

Nous examinerons successivement les travaux concernant les familles et sous-familles suivantes possédant un appareil buccal du type nématocérien: Phlebotominx, Ceratopogonidæ, Simuliidx, Blepharoceridx, Tabanidx, Rhagionidæx. Nous ne parlerons que de l'appareil buccal des femelles d'espèces pratiquant l'hématophagie, puisque, chez les mâles de ces espèces, les mandibules sont absentes ou réduites ou ne présentent pas de dispositifs morphologiques. permettant de pratiquer une lésion.

\section{Phlebotominæ}

La première étude de l'armement buccal de ces insectes est due à Grassi (1907): les mandibules sont brièvement décrites et les figures de ces pièces ne montrent aucun dispositif coaptatif comparable à celui qui sera décrit ultérieurement chez les Ceratopogonidæ et les Phlebotominæ. L'auteur a représenté (fig. 18, pl. 1) une coupe transversale du proboscis qui correspond à ce qui sera décrit par la suite : le labre présente, sur sa face inférieure, une gouttière que vient fermer une mandibule, les deux mandibules étant disposées à plat l'une au-dessus de l'autre et surmontant l'hypopharynx ; les maxilles sont disposées latéralement. Ce faisceau de pièces est placé dans la gouttière labiale.

Newstead (1911) a donné, lui aussi, une description des pièces buccales de Phl. papatasii, mais, en ce qui concerne les mandibules, il se contente de noter :

\& The mandibles are broad and blade-like, and have the outer edges faintly serrated, the serrations being rather widely separated. When at rest they lie apparently, superimposed one over the other. 》

Au cours de l'année 1926 paraissent trois études sur l'appareil buccal des Phlébotomes. Ce sont celles, très importantes, de Adler et Theodor, de Christophers, Shortt et Barraud, et enfin celle, beaucoup plus réduite, de Nitzulescu. Il est possible que la publication. 
de Adler et Theodor, qui porte la mention « reçu pour publication le 27 novembre 1927 , ait la priorité. Ces trois publications ont trait d'ailleurs à trois espèces différentes. En ce qui concerne la disposition des pièces constituant le proboscis, elles ne font que confirmer la description de Grassi. Pour ce qui est plus précisément des mandibules, Adler et Theodor ne décrivent, ni ne figurent, chez Phl. papatasii, aucun dispositif assurant la coaptation des mandibules entre elles, ou entre celles-ci et le labre ou l'hypopharynx. Ils décrivent en détail la musculature et arrivent, d'après leurs observations in vivo sous le microscope, à une interprétation du fonctionnement :

«Wen the mouth parts are in action the mandibles are rapidly abducted and adducted through a narrow angle and are at the same time rotated movements which can be readily followed in vivo under the microscope... During the act of feeding the mandibles no longer interpose between the epipharynx and hypopharynx and the two latter coming into opposition form the food canal, their teeth interlock and probably act as a strainer, preventing particles of too large a size from entering the food canal.

Christophers, Shortt et Barraud sont les premiers à mentionner, chez Phl. argentipes, l'existence d'une dépression médiane sur la mandibule. Nous ne pouvons mieux faire que de reproduire leur description et la figure (fig. $1 \mathrm{~A}$ ) qu'ils donnent :

« About the middle of the blade is a peculiar deformity or tache looking as though an injury had been inflicted by the dissecting needle but this is an entirely natural feature of the organ and is always present, being especially conspicuous in stained preparations. At this point the surface of the chitin appears to be depressed and it is possible that it has some actual function.»

Ces auteurs n'interprètent donc pas cette dépression comme un organe de mise en place, mais font cependant remarquer que rien n'assure la cohésion de ces pièces :

\& ....It is also remarkable that there seems to be so little to guard against dislocation of these organs, the chief piercing weapons presumably of the proboscis, which give the apparence of being very loosely articulated... »

Nitzulescu (1926) donne un schéma de la mandibule que nous reproduisons ici (fig. $1 \mathrm{~B}$ ) et qui montre, au milieu de la lame, une plage allongée ressemblant tout à fait à ce que Jobling décrira plus tard, en 1928, chez Culicoüdes, mais, dans le texte, Nitzulescu ne 


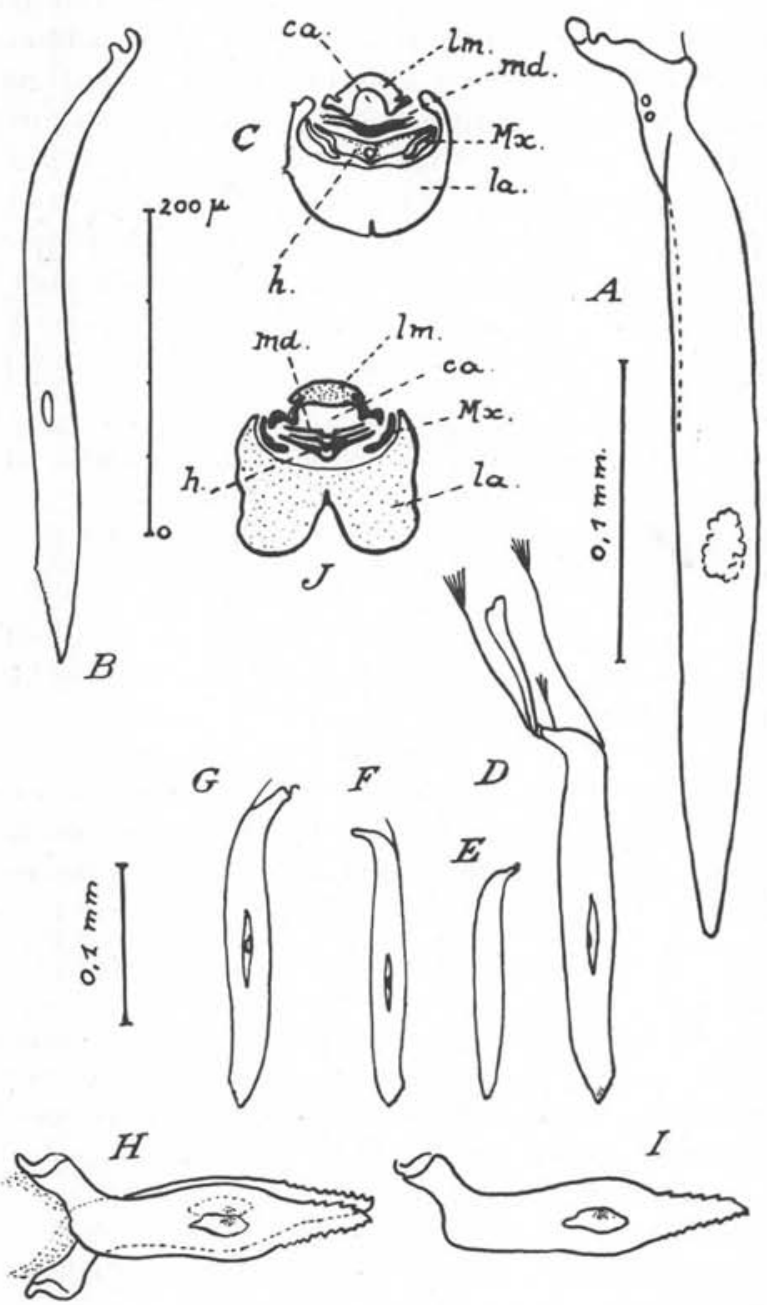

Fig. 1. - A) Mandibule de Phlebotomus argentipes, d'après Christophers et collab. ; B) Mandibule de Phlebotomus d'après Nitzulescu (1926) ; C) Coupe transversale des pièces buccales de Phlebotomus major, $\times 600$, d'après Jobling (1928) ; D) Mandibule de Culicoides pulicaris 우 ; E) C. pulicaris $\delta$; F) C. obsoletus $q$; G) id., C. vexans $q$ (fig. D à G d'après Jobling) ; H et I) Mandibule de Prionognathus marmoratus , d'après Carter et Macfie (1921) ; J) Coupe transversale, dans leur région médiane, des pièces buccales de C. vexans $q \times 540, c a$ : canal alimentaire, $h$ : hypopharynx, $l a: l a b i u m, l m$ : labre, $m d$ : mandibule, $M x$ : galea maxillaire. 
mentionne pas cette particularité. En outre, dans son interprétation du fonctionnement des mandibules, il a peut-être envisagé l'idée d'un fonctionnement en « ciseaux », sans toutefois prononcer ce nom puisqu'il écrit :

« L'extrémité de la mandibule n'est dentelée que sur un seul còté, le côté interne. Mais, quand les deux mandibules se superposent, elles forment comme un organe à extrémité unique, lancéolé et dentelé sur ses deux côtés comme dans la figure 4. Comme les dents de chaque mandibule sont placées sur le còté interne, ces deux organes coupent en croisant l'une sur l'autre (souligné par nous). Nous pouvons nous en rendre compte en examinant les muscles. De ceux qui actionnent les mandibules, le plus fort est l'adducteur, qui presse le bord dentelé dans les tissus. »

Jobling (1928), dans sa minutieuse étude consacrée au Cératopogonide Culicoides pulicaris, mentionne rapidement ce qu'il a observé chez Simulium ornatum et Phlebotomus major. Pour ce dernier, il donne le dessin d'une coupe passant par le milieu des pièces buccales, coupe montrant la disposition au repos. Nous la reproduisons fig. 1 C. Jobling écrit (p. 222) que, chez la femelle, les mandibules présentent aussi une gouttière médiane comme chez Simulium, et qu'il existe une trace du processus sur la surface ventrale membraneuse de la mandibule droite, qui occupe une position dorsale par rapport à la gauche. La trace de ce processus est visible aussi sur la préparation in toto de la mandibule droite et elle offre l'aspect d'une tache brunâtre.

Ainsi donc, les observations de Jobling sont en accord avec celles de Christophers, Shortt et Barraud, et avec la figure donnée par Nitzulescu. Par contre, ni Grassi, ni Adler et Theodor, ni beaucoup plus tard Snodgrass $(1943$, p. 52), ne font allusion à ce dispositif qui parait annoncer celui beaucoup plus perfectionné que nous allons retrouver chez les Cératopogonidés. Cependant, étant donné la qualité des observateurs qui n'ont pas signalé cette structure chez les Phlébotomes, on peut penser, soit que leur attention n'a pas été attirée par un tel dispositif, ou que celui-ci n'existe vraiment pas chez les espèces observées par eux. Nous ferons remarquer que l'état rudimentaire d'un tel appareil de mise en place peut s'expliquer par le fait que, chez les Phlebotominæ (comme chez les Culicidæ où ce dispositif est absent), le labium est très engainant et que la cohésion du faisceau vulnérant est assurée par la profonde gouttière labiale qui « conduit » celui-ci sur toute sa longueur. 


\section{Ceratopogonidæ}

L'étude morphologique des pièces buccales de ces Diptères a été faite par Carter, Ingram et Macfie (1920), Carter et Macfie (1921), Goetghebuer (1923), Léon (1924), et enfin Jobling (1928) qui leur a consacré une étude extrêmement minutieuse.

Carter et coll. (1920), dans leur rapide description de Culicoides sp. n., représentent notamment les mandibules, mais ne mentionnent pas l'existence d'un dispositif médian de mise en place. Par contre, Carter et Macfie (1921) décrivent, chez Prionognathus marmoratus sp. $\mathrm{n}$. femelle, les deux mandibules posées à plat l'une au-dessus de l'autre, et leurs figures 1 (a et b), reproduites ici (fig. 1, H et I), montrent qu'ils ont constaté l'existence d'une zone centrale, dont ils ne parlent d'ailleurs pas dans leur texte.

Jobling (1928, p. 220), à qui ont dû échapper les figures de Carter et Macfie, ainsi que les observations de Christophers et coll. sur les Phlébotomes, est le premier à donner une interprétation concernant ce dispositif :

"In the middle part of each mandible there is a very interesting structure, which is seen in cleared specimen as a median depression (d) outlined in the form of a spindle with a darkly pigmented round or ovoid body in the middle or slightly at the side (cf. fig. 1, D a G, d'après Jobling). So far I know, the structure and functions of the depression and the associated parts have never been correctly understood, and since they are directly related to the position of the mandibles in relation to the other mouth parts, it will be best if they are considered together with the latter. When the mouth parts are at rest, the mandibles are closed like scissors and their saw-like edges slightly project beyond the antero-lateral portions of the labrum-epipharynx, below the lateral teeth (text fig. 1 A). In transverse sections of the mouth parts (pl. XI; fig. 14-19) it is seen that the mandibles occupy the space between the labrumepipharynx and the hypopharynx. The left mandible always lie above the right, which is tightly applied to the hypopharynx. As the anterior part of the mandible is bent inwards, the saw-like edge of the right mandible is on the left and that of the left mandible on the right side of the labrum-epipharynx.

The above disposition of the mandibles is maintened by the conformation of their middle parts on the following manner. The dorsal surface of each mandible has a median depression outlined in the form of a spindle, as already mentioned, and under this depression, but on the ventral surface, there is a strongly chitinized process (pl. X, fig. 11, pl. XI, fig. 18) which in the left mandible is slightly broadened ventrally and is interlocked with the depression on the dorsal surface of the 
right mandible. The process of the latter is much narrower than that of the left mandible and is interlocked with the lumen of the salivary duct of the hypopharynx. In some transverse sections of the mouth parts the mandibles and the hypopharynx fit each other so tightly that there is no space left between their adjacents parts. »

Ce texte ne laisse donc aucun doute: pour Jobling, les deux mandibules sont verrouillées par le dispositif de mise en place qui les rend solidaires, et les verrouille également avec la gouttière salivaire de l'hypopharynx qui est ainsi transformée en un canal (cf. fig. $1 \mathrm{~J}$ ).

Après avoir étudié la musculature des mandibules, Jobling, qui a observé sous la loupe binoculaire des femelles de Culicoïdes en train de se nourrir, en arrive à l'explication du mécanisme de la piqûre. Pour lui, le labre, l'hypopharynx, et les mandibules forment ensemble un stylet perforant qui exécute des mouvements d'avant en arrière pendant la piqûre. Lorsque ce stylet composite a pénétré dans la peau d'un peu plus de la moitié de sa longueur, les mouvements de protraction et de rétraction cessent. Quand s'effectue l'aspiration du sang, les mandibules ne s'écartent pas vers l'extérieur (ainsi que Adler et Theodor l'admettent pour les Phlébotomes), mais restent accolées grâce à leur dispositif de mise en place, et le canal alimentaire est bien formé par la gouttière ventrale du labre fermé par la mandibule gauche, ce qui avait déjà été constaté par Léon (1924).

En plus de ces mouvements d'ensemble, effectués avec le labre et l'hypopharynx, les mandibules peuvent, dit Jobling, subir grâce à leur propre musculature des mouvements d'abduction et d'adduction, mais ce mouvement est limité par la longueur du sillon dans lequel le processus de la mandibule gauche $\epsilon$ st engagé.

Les galea maxillaires, faiblement pigmentées et difficiles à observer in vivo, effectueraient seulement, si l'on en juge d'après leur musculature, des mouvements de protraction et de rétraction. Le labium sert à maintenir les autres pièces en position au cours de la piqûre.

Snodgrass (1944, p. 56), qui a retrouvé ce mème dispositif coaptatif des mandibules chez Culicoides furens (Poey), conclut, d'après l'étude de leur musculature, qu'en dépit de leur aspect, il est improbable que les mandibules puissent travailler comme des ciseaux. 


\section{Simuliidæ}

Des observations anciennes ont été faites sur les pièces buccales des Simuliidæ (Smith, 1890 ; Cragg, 1913 ; Emery, 1913 ; Cameron, 1922 ; Blacklock, 1926). En ce qui concerne le fonctionnement des mandibules, le premier travail à citer est celui de Nitzulescu (1926), généralement passé sous silence dans la bibliographie de langue anglaise.

Cet auteur, qui a pratiqué des coupes histologiques (fig. 2, C et D) dans le proboscis de Simulium, a montré que les mandibules sont interposées, à plat, l'une au-dessus de l'autre, entre le labre et l'hypopharynx. Il a vu aussi que la mandibule inférieure ferme le canal de l'hypopharynx, alors que la mandibule supérieure ferme la gouttière ventrale du labre qui devient ainsi le canal alimentaire. Pour lui, cette disposition n'est pas seulement réalisée au repos, mais aussi pendant l'acte de piqûre et l'aspiration de sang : comme principale preuve, il invoque l'existence d'une petite saillie ( $t m$, fig. $2 \mathrm{C}$ ), existant sur la face supérieure (souligné par nous) de chaque mandibule et destinée à s'engrener avec le labre, cet engrenage assurant une union plus étroite entre les pièces.

A la suite de la publication du travail fondamental de Jobling (1928), Nitzulescu (1928) revient sur cette question et désigne ce dispositif de mise en place sous le nom de « tubérosités mandibulaires ». Il fait remarquer que, contrairement à ce qui a été observé par Jobling chez Culicoides, ce dispositif, chez Simulium, est dorsal et non pas ventral. Nitzulescu ajoute aussi que Jobling ne cite pas son travail, bien que la figure que celui-ci donne d'une coupe transversale des pièces buccales de Simulium soit absolument comparables à celle que lui, Nitzulescu, a publiée antérieurement. La figure de Jobling révèle en effet la présence de ces tubérosités, mais l'auteur anglais, qui n'a pas décrit l'aire claire, pourtant bien visible (fig. $2 \mathrm{E}$ ) au milieu de chaque mandibule, n'a pas interprété ces formations comme un dispositif de mise en place et se contente d'écrire :

\& The mandibles of Simulium are strongly guttured ventrally, their ventral surface being membranous and devoid of process. »

Smart (1935) constate l'existence, au centre de chaque lame mandibulaire, d'une aire claire déprimée, semblable à celle trouvée chez Culicoides par Jobling en 1928.

Avec Gibbins (1938), le dernier pas est franchi, cette dépression claire va être assimilée purement et simplement à un « axe »du 


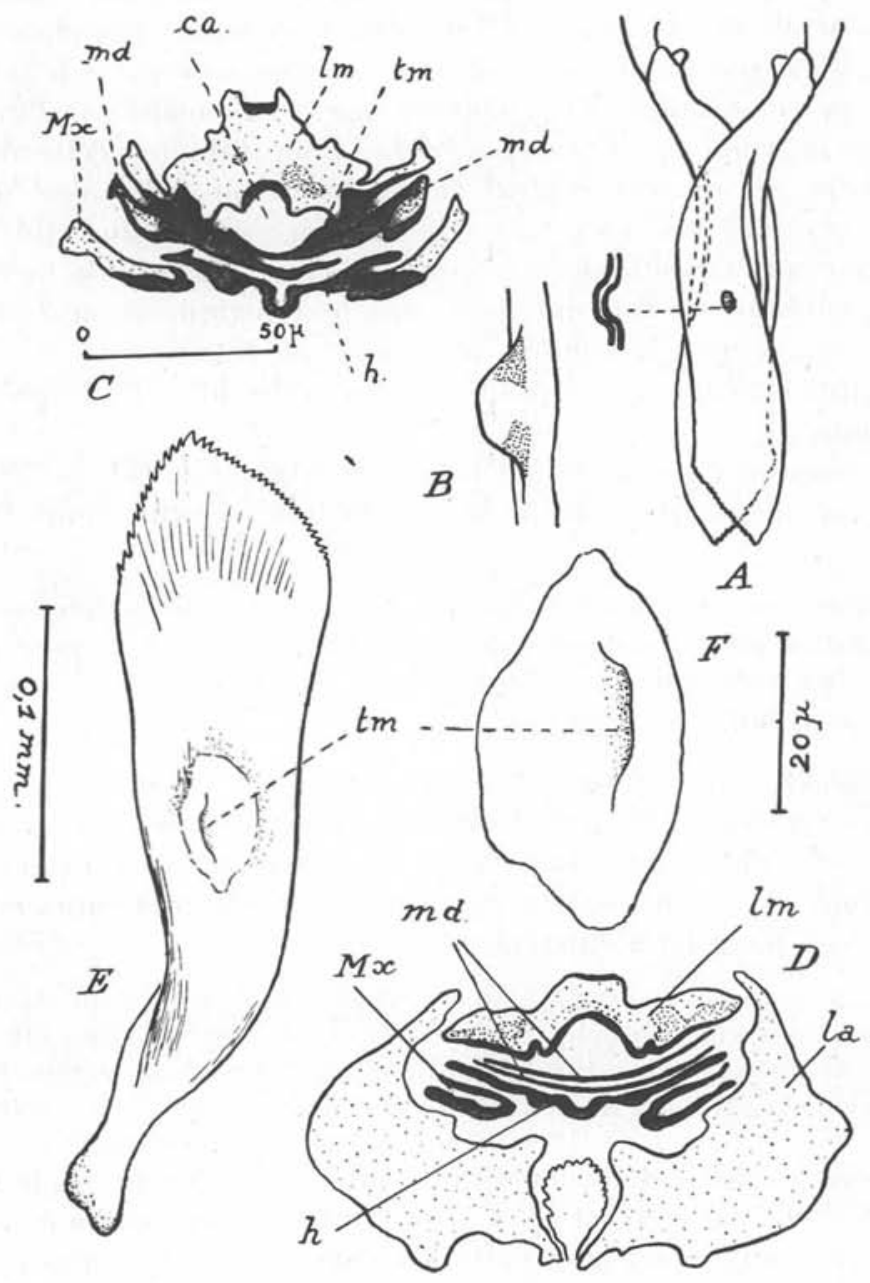

FIG. 2. - Simuliider : A) Mandibule de Simulium damnosum, d'après GıBвiss.

B) Vue latérale du processus médian de la mandibule, d'après GrbBins. C) Simulium sp., coupe transversale des pièces buccales (le labium n'est pas représenté) dans la moitié postérieure, exactement au niveau des « tubérosités mandibulaires \$ (lm), d'après Nrtzulescu. D) Simulium buissoni, coupe transversale des pièces buccales, dans la moitié antérieure, d'après Nrtzulescu. E) Mandibule gauche de $S$. ergthrocephalum (de Geer) $q$ (fig. originale). F) id., « tubérosité mandibulaire » fortement grossie (pour l'explication des figures, ef. fig. 1). 
mème type que celui décrit par Jobling chєz Culicoides, bien que cet auteur ait déclaré 'qu'un tel dispositif n'existait pas chez Simulium et bien que Nitzulescu et Jobling (si l'on se reporte à la figure donnée par ce dernier) aient montré que ce dispositif est d'un type différent de celui des Ceratopogonidæ. Pour Gibbins, cette dépression claire est un axe central en forme de protubérance, autour duquel pivotent les deux lames de la paire de ciseaux qui, entre leurs extrémités denticulées, pratiquent l'incision initiale lors de la piqûre. Gibbins n'a fait aucune coupe histologique et ne fait allusion ni aux coupes de Jobling, ni à celles de Nitzulescu.

Edwards (1939, p. 52) reproduit, sans la discuter, l'interprétation de Gibbins.

La première critique est faite par Snodgrass (1944, p. 58), qui décrit les mandibules de $S$. venustum Say comme (nous traduisons):

«Posées l'une sur l'autre, la gauche sur la droite, et maintenues dans cette position par un dispositif de verrouillage (interlocking mechanism) comme chez Culicoides, de telle sorte qu'elles ressemblent de façon frappante à une paire de ciseaux. »

Snodgrass admet done que ce dispositif est du même type que celui des Culicoides et ne fait allusion ni à Nitzulescu, ni à Jobling. Cependant, l'idée d'un fonctionnement en ciseaux ne lui paraît pas acceptable, car la musculature qui actionne ces prétendus ciseaux est du type habituel abducteur-adducteur...

«...And does not appears in any way adapted to giving the mandibles a scissor movment on their interlocking mechanism, Jobling (1928), as already noted, says that the mandibles of Culicoides remain locked together during the act of puncturing the skin (*). »

Nicholson (1945), dans son étude comparative des pièces buccales de $S$. (E.) dacotense D. et Sh. (= lascivum Twinn) et de S. venustum Say, montre que, chez cette dernière espèce, le dispositif médian correspond à la description de Nitzulescu : la protubérance est bien dorsale (et non ventrale comme le dit Gibbins) et « s'accroche » au bord de la gouttière labrale. En outre, chez $S$. dacotense qui n'est pas piqueur, il n'existe, au milieu de chaque mandibule, qu'une aire claire, qui n'est, suivant Nicholson, ni une dépression, ni une saillie. Cette dernière constatation montre bien, à notre avis, l'importance de ce dispositif en ce qui concerne l'acte de piqûre, puisqu'il fait défaut chez une espèce non piqueuse.

(*) Квағсніск (1942) a étudić $S$. lascivum Twinn, mais il s'agit d'une espèce non hématophage. 
Malgré ce travail donnant raison à Nitzulescu, les auteurs qui se sont intéressés par la suite à cette question se sont contentés de citer Gibbins et Snodgrass, ou tout au moins n'ont fait aucune allusion aux descriptions pourtant exactes de Nitzulescu et Nicholson.

Wanson (1950) parait admettre les idées de Gibbins puisqu'il écrit (p. 697): «Les mandibules superposées coupent, en se croisant l'une sur l'autre, par leur extrémité distale dont le bord interne seulement porte des dents. 》

J'ai moi-même, en 1953 (p. 20), fait état des objections de Snodgrass cité plus haut et fait remarquer que $S$. damnosum étudié par Gibbins est une des rares espèces présentant un prétendu « axe » mandibulaire à peu près circulaire, qui pourrait donc permettre un mouvement de ciseaux, alors que, chez la grande majorité des espèces, cet « axe » est en réalité fusiforme ou allongé. Pour cette raison, j'ai considéré alors ce dispositif comme un dispositf de «mise en place ». A cette époque, j'admettais encore la description de Gibbins et la superposition de ces deux soi-disant dépressions, car la publication de Nitzulescu et la brève remarque de Jobling m'avaient échappé, mais l'idée d'un fonctionnement " en ciseaux » ne me paraissait pas acceptable.

Freeman et de Meillon (1953, p. 13) se contentent de citer, sans commentaire, l'interprétation de Gibbins et celle de Snodgrass sans mentionner Nitzulescu, ni le travail de Nicholson, ni nos propres observations.

Rubtzov, enfin (1956, p. 21), donne une figure schématique d'une coupe transversale des pièces buccales, correspondant à l'ancienne interprétation : canal alimentaire formé par accolement du labre et de l'hypopharynx, les mandibules étant placées latéralement audessous de ce dernier et les maxilles au-dessous des mandibules. Sa figure $4 \mathrm{D}$ représente une mandibule, avec, au milieu de celle-ci, le prétendu «axe». Au point de vue du fonctionnement, Rubtzov ne parle pas du fonctionnement en ciseaux, mais écrit que les mandibules, agissant de haut en bas, scieraient l'incision initiale effectuée par le labre, les mandibules déchirant ensuite, par un mouvement inverse, les tissus et les vaisseaux sanguins.

Ainsi donc, en ce qui concerne le fonctionnement des mandibules chez les Simulies, nous sommes en présence de deux explications : celle de Nitzulescu-Nicholson et celle de Gibbins qui, malgré certaines critiques, parait acceptée. C'est pourquoi nous avons repris l'examen des préparations de pièces buccales que nous possédons dans nos collections, afin de vérifier quel est le dispositif 

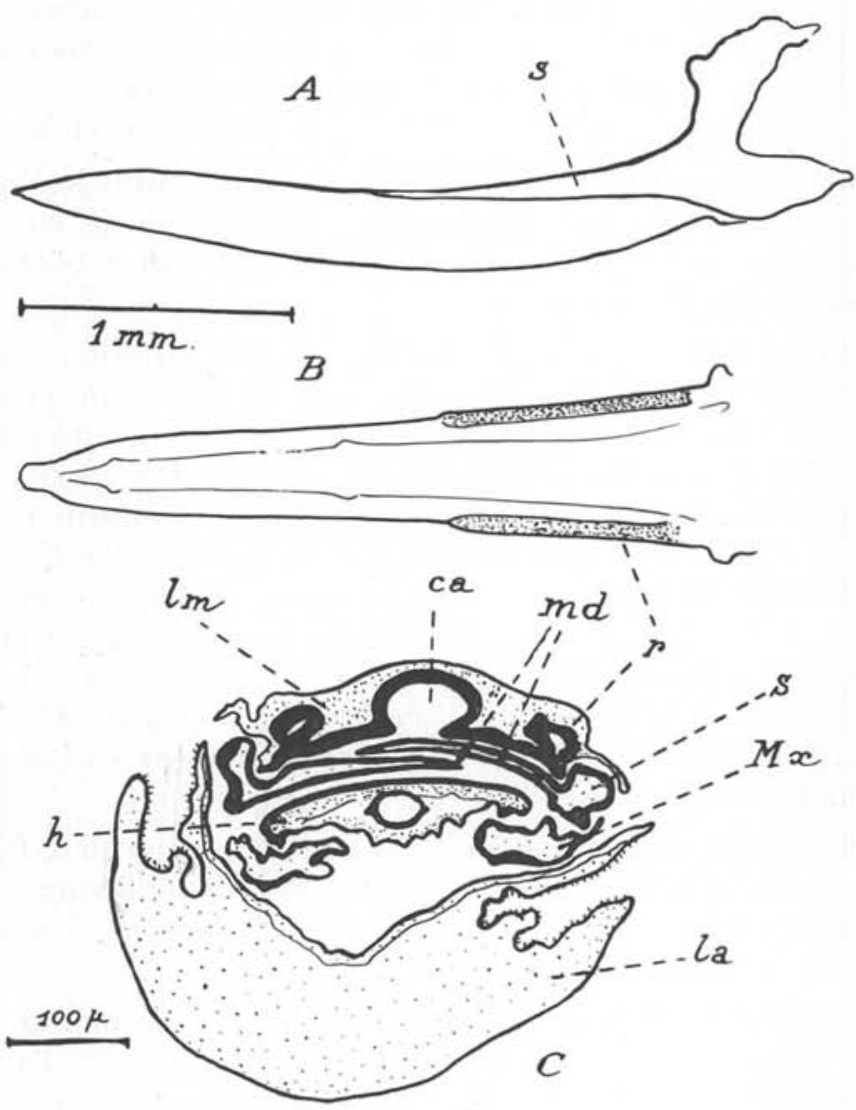

F1G. 3. - Tabanidze. A) Mandibule de Tabanus sp. ‥ B) Labium du mème exemplaire. C) Coupe transversale des pièces buccales chez Hæmatopota pluvialis, d’après Nitzulesce.

morphologique existant réellement. L'aspect que nous avons toujours observé est celui décrit par Nitzulescu et il n'est pas nécessaire pour s'en convaincre de refaire de nouvelles coupes histologiques (celles de Nitzulescu et de Jobling concordent d'ailleurs parfaitement) ; l'examen des mandibules à un fort grossissement prouve que les « tubérosités mandibulaires » sont bien placées sur la face dorsale de chaque mandibule, au milieu de l'aire claire; chacune de ces tubérosités apparait, en fait, comme une petite glissière tournée vers l'extérieur et qui vient s'accrocher à la bordure interne de la gouttière ventrale du labre. Cet aspect est représenté sur nos 
figures $2 \mathrm{E}$ et $\mathrm{F}$ qui correspondent à l'aspect observé chez $S$. reptans L. et $S$. erythrocephalum de Geer. Les deux mandibules ne sont donc pas verrouillées l'une à l'autre, comme elles le sont chez Culicoides, mais chacune est accrochée séparément au labre. L'action des deux puissants muscles adducteurs décrits par les auteurs (cf. fig. $24 \mathrm{~K}$, in Snodgrass) a donc pour effet, pendant la piqûre, d'appliquer fortement la glissière $t m$ contre la crête correspondante du labre; ainsi est réalisé un dispositif vulnérant doué d'une grande cohésion pendant les mouvements de bas en haut.

De plus, si l'on examine les pièces buccales in toto, on constate aisément que les zones claires des mandibules ne se superposent pas, mais sont largement écartées.

Quant à la coaptation des mandibules et de l'hypopharynx, elle ne peut être réalisée comme le décrit Gibbins, puisque les tubérosités sont dorsales et non pas ventrales. Par contre, un coup d'œil jeté aux coupes transversales, en particulier à la figure 13 , pl. X, de Jobling, montre que chaque mandilule porte vers l'extérieur une rainure (v. fig. $4 \mathrm{D}$ ), dans laquelle vient se placer, à droite et à gauche, l'arête latérale de l'hypopharynx. La cohésion du faisceau labre-mandibules-hypopharynx est ainsi réalisée par un système plus complexe que celui existant chez Culicoides et probablement aussi efficace, mais complètement différent de celui décrit par Gibbins à la suite d'observations un peu superficielles.

Bien que les mouvements des pièces buccales au cours de la piqûre n'aient pu être observés chez les Simulies comme ils l'ont été, par Gordon et ses coll., chez Aedes aegypti, Cimex et Glossina, il semble difficilement concevable qu'un tel faisceau vulnérant aussi cohérent, constitué de larges lames, puisse « rechercher 》 un capillaire sanguin et assurer une nutrition capillaire. Il semble très probable que la nutrition s'effectue exclusivement à partir d'un microhématome (pool feeding), tel que celui qui a été décrit chez le Tabanide Chrysops par Gordon et Crewe (1948 et 1953) ; ce microhématome se forme à la suite de la large dilacération tissulaire et vasculaire qui a pour effet de déloger du même coup les microfilaires dermiques (Onchocerca). Wanson (1950, p. 697) a d'ailleurs constaté, sur coupes histologiques, l'épanchement du sang en un «lac minuscule » au voisinage de la lésion produite par les pièces buccales de $S$. damnosum. 


\section{Blepharoceridæ}

Chez ces Nématocères, les femelles sont prédatrices surtout d'Insectes, et celles de quelques espèces (g. Curupira) pratiqueraient l'hématophagie aux dépens de Vertébrés. Une étude des pièces buccales a été faite par Vimmer (1924) (travail que nous n'avons pu consulter).

Snodgrass (1944, p. 61) écrit que l'armement buccal ressemble à celui des Simuliides, mais que les mandibules ne sont pas verrouillées (interlocked). Les quelques préparations que nous possédons nous ont permis de constater que les manäibules, fortement dentées sur leur bord interne et sur presque toute la longueur de celui-ci, sont effectivement posées à plat l'une sur l'autre au-dessous du labre. Mais nous n'avons pu observer s'il existe un dispositif rappelant celui des Phlébotomes ou des Simuliides.

\section{Tabanidæ}

Chez les Tabanidæe, l'appareil buccal est encore du type nématocérien. Snodgrass (1944, p. 61 et suivantes) a repris la description de cet appareil et, récemment, Gordon et Crewe (1953) ont apporté des précisions fort intéressantes sur le mécanisme de la piqûre chez Chrysops. Dans cette dernière publication, les auteurs annoncent une étude morphologique prochaine des pièces buccales par Lavoipierre. Snodgrass (loc. cit.), en ce qui concerne les mandibules, ne mentionne pas l'existence d'un dispositif de coaptation et la coupe transversale de l'appareil qu'il donne est une reproduction, d'après Vogel (1921), d'une section passant au niveau des labelles. Cependant, dans une publication antérieure de Nitzulescu (1926), un tel dispositif coaptatif est décrit et la coupe illustrant ce travail ne laisse aucun doute (fig. $3 \mathrm{C}$ ). La coaptation est obtenue par un dispositif différent de celui qui est réalisé chez les Cératopogonidés, mais rappelant ce qui existe chez les Simuliides : dans sa moitié proximale, en effet, chaque mandibule porte un repli latéral dorsal (S), qui vient s'engrener avec le bord latéral du labre.

Nous avons repris nos préparations in toto de pièces buccales de Tabanus et Haematopota, et nous avons constaté que ce repli formant " rainure de guidage * existe bien et qu'il est très visible (S, fig. $3 \mathrm{~A}$ ). En outre, le labre porte latéralement un renforcement chitineux (r, fig. $3 \mathrm{~B}$ ), qui a sensiblement la même longueur que 

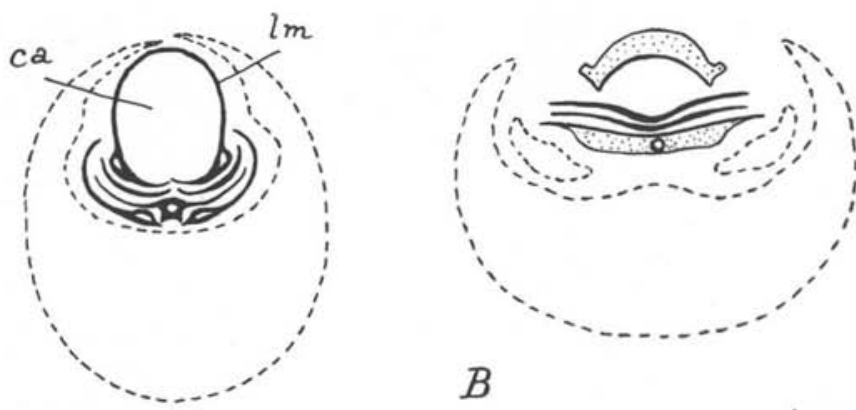

$B$

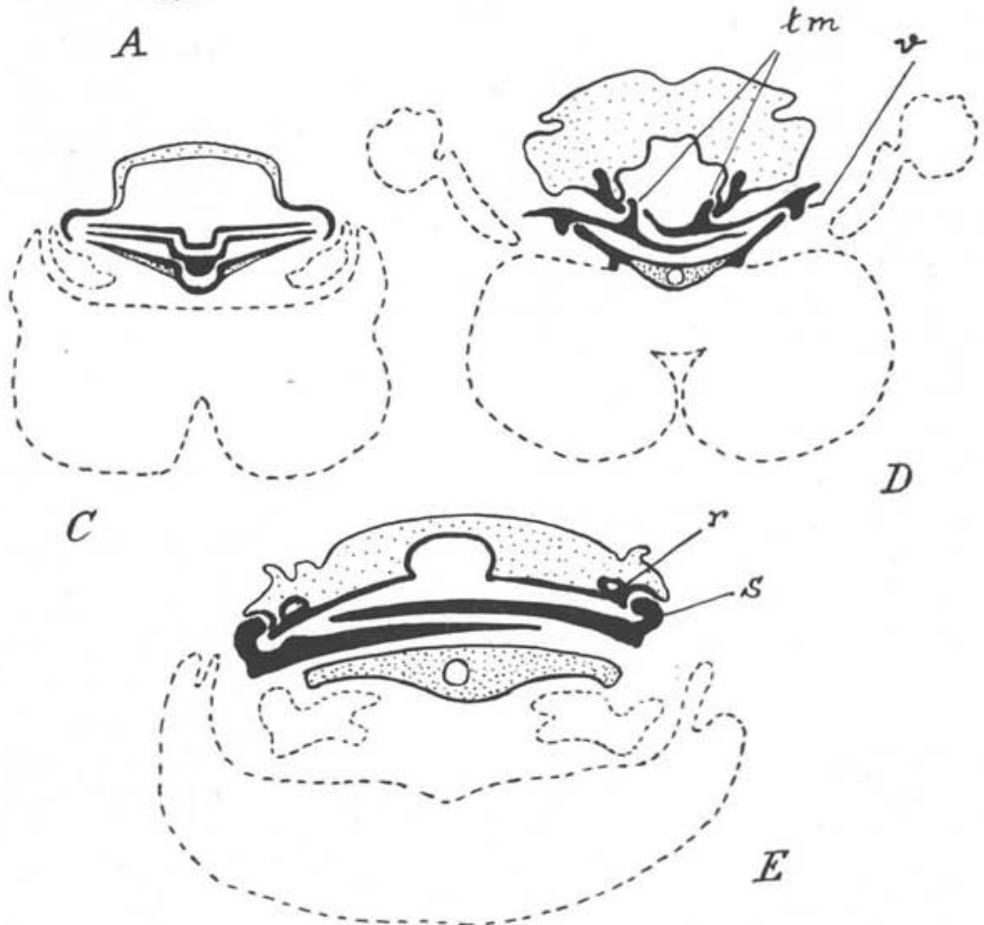

Fıc. 4. - Coupes transversales schématiques des pièces buccales montrant les dispositifs assurant la mise en place des mandibules avee le labre et l'hypopharynx : A) Type labral (Culicide) ; B, C, D, E) Type labro-inandibulaire : $\mathrm{B}$ et C) Sous-type Phlebotomina - Ceratopogonida :

B) Phlebotomus, C) Culicoides ; D) Sous-type Simulium, E) Sous-type Tabanus. 
ce repli mandibulaire. Il est d'ailleurs curieux de constater que ce repli est bien représenté sur les figures, déjà anciennes, que l'on retrouve chez des auteurs tels que Patton et Cragg (1913, fig. 4, pl. IV), Patton et Evans (1929, fig. 184, p. 308), Snodgrass (loc. cit., fig. $25 \mathrm{~F}$, p. 63), Matheson (1950, p. 425, fig. 157).

\section{Rhagionidæ (Leptidæ)}

Cette famille, composée surtout de prédateurs d'Insectes, renferme cependant quelques espèces pratiquant l'hématophagie aux dépens de Vertébrés. Celles-ci appartiennent aux genres Symphoromyia Frauenfeld, aux U.S.A. (Californie), et Spaniopsis White, en Australie (Tasmanie). En France, Séguy (1926) cite Rhagio scolopaceus L. et Rh. strigosus $\mathrm{Mg}$. comme attaquant les Vertébrés. Enfin, Desportes (1941) a observé Atrichops crassipes Mg. se gorgeant de sang sur la grenouille.

L'appareil buccal de $S$. atripes Bigot est décrit par Snodgrass (loc. cit., p. 65) comme ressemblant à celui des Tabanida; cet auteur ne mentionne aucun dispositif coaptatif des mandibules et la figure $26 \mathrm{C}$, qui représente une mandibule, ne fait apparaître l'existence ni d'un repli latéral comme celui des Tabanidés, ni d'un dispositif dorsal médian comme il en existe chez Simulium. Aucune coupe transversale du faisceau vulnérant n'est donnée dans le travail. L'étude précise de ces organes chez les Rhagionidæx serait peut-être à reprendre.

\section{Conclusions}

D'après les remarques que nous venons de formuler, il semble que, chez les femelles de Diptères, Nématocères et Brachycères hémophages, il est difficile d'admettre que les mandibules puissent fonctionner à la façon des ciseaux, cette idée lancée par Gibbins pour Simulium damnosum étant fondée essentiellement sur la description inexacte d'un axe mandibulaire de rotation, " axe » qui se révèle, en fait, être un simple dispositif de mise en place assurant la cohésion du labre et des lames mandibulaires.

L'étude des représentants des familles et sous-familles suivantes : Culicidx, Phlebotominæ, Ceratopogonidæ, Simuliidæ, Tabanidæ $\left(^{*}\right)$, a permis de mettre en évidence divers types de dispositifs

(*) La description des pièces buccales de Blepharocerida nous parait trop incomplète pour que nous puissions actuellement la mentionner. 
assurant la cohésion du faisceau de stylets. Il semble que l'on puisse distinguer, suivant la constitution du canal alimentaire et le dispositif coaptatif existant :

$1^{\circ}$ Le type labral (ou type Culicide), dans lequel le canal alimentaire est constitué entièrement par le labrum formant un véritable canal, par l'incurvation prononcée de ses parties latérales. La cohésion de toutes les pièces constituant le faisceau est réalisée sans coaptation particulière, toutes les pièces étant étroitement emprisonnées dans une gouttière labiale presque close (fig. $4 \mathrm{~A}$ ).

2 " Le type labro-mandibulaire. - Ce type, décrit par Nitzulescu (1926) pour les Tabanides et les Phlébotomes, est réalisé chez les Phlebotomina, Ceratopogonidx, Simuliidx, Tabanidx et vraisemblablement chez les Rhagionidx. Dans ce type, le canal alimentaire est formé par une gouttière labrale largement ouverte vers la face ventrale. La fermeture de cette gouttière est assurée par l'application étroite, contre celle-ci, de la mandibule supérieure qui est placée à plat au-dessus de l'autre lame mandibulaire, celle-ci recouvrant elle-même l'hypopharynx et fermant ainsi la gouttière salivaire.

La "liaison » des mandibules au labre d'une part et à l'hypopharynx d'autre part est rendue nécessaire dans le type labromaxillaire, du fait que la gouttière labiale, largement ouverte dorsalement, n'enferme pas étroitement le faisceau, comme cela est réalisé dans le type labral. Suivant la position des dispositifs de mise en place, nous proposons de distinguer les sous-types suivants :

a) Le sous-type Phlebotomina-Ceratopogonida: Le dispositif coaptatif, qui est pleinement réalisé chez les Ceratopogonidæ, est placé dans la région médiane des mandibules qui sont superposées sur presque toute leur longueur ; chaque mandibule porte dorsalement une dépression, qui se traduit ventralement par un processus s'encastrant dans la dépression correspondante de la pièce sous-jacente, le processus ventral de la mandibule inférieure s'encastrant en mème temps dans la gouttière salivaire de l'hypopharynx. Si la liaison mandibules-hypopharynx est ainsi réalisée, ce dispositif n'assure pas la liaison du labre avec le faisceau constitué par ces trois lames. Or, si on se reporte à la coupe transversale de Culicoides d'après Jobling, on constate que les bords latéraux du labre sont renforcés, formant une gouttière tournée vers l'intérieur; dans cette rainure paraissent s'encastrer ces trois lames (md. + hypoph.) (fig. 4 B et C).

Ce sous-type parait seulement ébauché chez les Phlebotominæ. 
b) Le sous-type Simulium (fig. 4 D) : Les mandibules sont disposées à plat l'une au-dessus de l'autre, mais ne se superposent vraiment que dans leur région terminale. Bien que le dispositif de mise en place des mandibules, qui est situé dans la région médiane de chacune de celles-ci, se présente, à un examen rapide, comme celui des Cératopogonidés, ce dispositif est très différent puisqu'il est dorsal, comme l'ont vu Nitzulescu, puis Nicholson ; chaque petit ergot $(\mathrm{tm})$ s’accroche au bord intérieur de la gouttière labrale ; en outre, la liaison mandibules-hypopharynx est réalisée par un dispositif du même type $(v)$, mais porté par la face latéro-ventrale de chaque mandibule. De tels dispositifs, ainsi que le type de musculature actionnant les mandibules, rendent très improbable le fonctionnement en « ciseaux " décrit par Gibbins.

c) Le sous-type TABANIDA (fig. $4 \mathrm{E}$ ) : La coaptation labre-mandibules rappelle le type précédent, mais le dispositif d'accrochage est placé sur le bord externe de chaque mandibule et dans la moitié proximale de celle-ci. Aucun dispositif assurant la liaison mandibule-hypopharynx ne parait exister.

Ainsi donc, le sous-type Simulium parait intermédiaire entre le sous-type Ceratopogonidx et le sous-type Tabanidx. Ceci parait constituer un nouvel argument en faveur de la position systématique de la famille des Simuliidæ, classiquement placée dans le sous-ordre des Nématocères et même récemment, par E. Séguy (1951, p. 602), dans la super-famille des Culicoidea, première section (Simuliidæ, Ceratopogonidæ, Chironomidæ) de ce sous-ordre. Nous rappellerons que, personnellement, en accord avec les anciens systématiciens qui considéraient les Simuliidæx comme des Nemocera anomala ou des Paranématocères, nous pensons que de nombreux caractères font des Simuliides des Nématocères de transition annonçant les Brachycères.

Ces caractères sont :

a) l'aspect général du corps et des antennes ;

b) la disposition du système sympathico-endocrine de la larve.

Depuis nos remarques formulées en 1948 et 1953, d'autres constatations sont venues, nous semble-t-il, appuyer cette idée. Lewis (1952) rapporte les observations de Pringle (1948) sur les haltères de Simulium ( $S$. damnosum) : ces organes sont très développès pour des Nématocères; l'orientation en diagonale des sensilles (considérée comme nécessaire pour assurer la fonction gyroscopique), sur la plaque basale, présente une similitude remarquable avec ce qui existe chez un Muscidé ou un Syrphidé... Ces constata- 
tions de Pringle, fait remarquer Lewis, sont particulièrement intéressantes en ce qui concerne la similitude de l'aspect général qui existe entre les Simulïdæ et les Diptères supérieurs.

En ce qui concerne l'appareil buccal des Simuliida, on sait déjà qu'il évoque, par ses larges lames mandibulaires, ses maxilles, ses labelles considérablement développées, celui des Tabanidæ. Snodgrass $(1944$, p. 60$)$ a fait remarquer aussi que, chez les Simuliides, la pompe cibariale était très développée, mais que la pompe pharyngienne était beaucoup plus réduite que chez les autres Nématocères hémophages, et l'on sait que la pompe pharyngienne des TaLanidés (et des Asilidés) est d'un type très différent de celle des Nématocères.

L'étude des coaptations mandibulaires chez les Simuliides et Tabanidés que nous venons de faire montre que le dispositif réalisé chez la première famille est un type intermédiaire entre ce qui existe chez de véritables Nématocères comme les Ceratopogonidæ et les Phlebotominæ, et annonce déjà ce qui sera réalisé chez les Tabanidæ.

\section{BIBLIOGRAPHIE}

Ant.ER (S.) et Theodon (O.) (1926). - The mouth parts, alimentary tract and salivary apparatus of the female in Phlebotomus papatasii. Ann. Trop. Med. and Parasitol., $26: 109$.

Бт.Аскі.оск (D. В.) (1926). - The development of Onchocerca volvulus in Simulium damnosum. Ann. Trop. Med. and Parasitol., $20: 1$.

Cameron (A. E.) (1922). - The morphology and biology of a canadian cattle infesting blackfly Simulium simile Mall. Bull. Dept. Agric. Canada, new ser. $n^{\circ} 5$.

Carten (H. F.), Ingram (A.), Macfie (J. W. S.) (1920). - Observations on the Ceratopogonine midges of the Gold Coast with deseriptions of new species. Part II. Ann. Trop. Med. and Parasitol., 1/4(2) : 211-270. Idem, Part III, $1 /$ (3) : 309-331.

Cimistophens (S. R.), Shontт (H. E.) et Barmaud (A. J.) (1926). - The anatomy of the Sand-fly, Phlebotomus argentipes. Ann. and Brun. (Diptera), I, the head and mouth parts of the imago. Ind. Med. Res. Mem., $\mathrm{n}^{\circ} 4$, p. 177 .

Cragi (F. W.) (1913). - Studies on the mouth parts and sucking apparatus of some Diptera. Sci. Mem. Off. Med. and San. Dept. Govt. India, new ser. $\mathrm{n}^{\circ} 58$.

Cú́not (L.) (1925). - L'adaptation. Encycl. Entom., G. Doin édit., Paris.

Despontes (C.) (1941). - Nouvelles recherches sur la morphologie et sur l'évolution d'Icosiella neglecta (Diesing, 1851), filaire commune de la grenouille verte. Annales de Parasitologie, 1941, n 1-2-3, vol. XVIII, 46-67.

Edwands (F. W.) (1939). - "Simuliidæ», in British Blood sucking flies, par Edwards (F. W.), Oldroyd (H.) et Smart (J.). British Museum. 
EMERY (W. T.) (1913), - The morphology and biology of Simulium vittatum. Kansas Univ. Sci. Bull., $8: 323$.

Freeman (P.) et de Menllon (B.) (1953). - Simuliida of the Ethiopian Region. British Museum (Natural History), 224 pp.

(iibbins (E. G.) (1938). - The mouth parts of the female in Simulium damnosum Theobald, with special reference to the transmission of Onchocerca volvulus Leuckart. Ann. Trop. Med. and Parasitol., 82 (1) : 9-20.

Goethiebuen (M.) (1923), - Ceratopogonina de Belgique, parasites accidentels de l'homme. Bull. Soc. Entom. Belg., 3/:1.

Gordon (R. M.) et Crewe (W.) (1953), - The deposition of the efective stage of Loa loa by Chrysops silacea; and the early stages of its migration to the deeper tissues of the Mammalian host. Ann. Trop. Med. and Parasitol., $47: 74-85$.

- (1948). - The mechanism by which mosquitoes and tse-tse fllies obtain their blood-meal, the histology of the lesions produced, and the subsequent reactions of the Mammalian host; together with some observations on the feeding of Chrysops and Cimex. Ibid., $42: 334$.

Grassi (B.) (1907). - Recerche sui Flebotomi. R. Academia dei Lineri Roma.

Grexien (P.) (1949). - Contribution à l'étude biologique des Simuliides de France. Physiol. Comp. et Ecol., I, (1948), n 3-4, 165-330.

- (1953). - Simuliide de France et d'Afrique du Nord. Encycl. Entom., (A), 29, $170 \mathrm{pp}$.

Jobling (B.) (1928). - The structure of the head and mouth parts in Culicoides pulicaris L. (Diptera nematocera). Bull. Entom. Res., 18:211-226.

Leon (N.) (1924). - L'armature buccale et la pompe salivaire des Culicoides pulicaris. Bull. Sect. Sci. Acad. Roumaine, 9, (1-2) : 133.

LEwIS (D. J.) (1952), - Simulium damnosum and ist relation to Onchocerciasis in the Anglo-Egyptian Sudan. Bull. Ent. Res., 43, (4) : 597-644 (janvier 1953).

Matheson (R.) (1950), - Medical entomology, Comstock Publishing Company, Inc. Ithaca, New-York.

Nkwstbad (R.) (1911). - The Papataci flies of the Matese Islands. Ann. Trop. Med. and Parasitol., 5, (2) : 139-186.

Nicholson (H. P.) (1945). - The morphology of the mosth parts of the non biting blackfly Eusimulium dacotense D, et S., as compared with those of the non biting species, Simulium venustum Say. Ann. Entomol. Soc. Amer., $38: 281-297$.

Nrtzulescu (V.) (1926). - Sur la constitution du canal alimentaire chez les Plébotomes. Bull. Soc. Path. exot., $19: 709-714$.

(1926). - Sur l'armature buccale des Tabanides. C.R. Soc. Biol. (Paris), $95: 1152-54$.

(1926). - Contribution à l'étude de l'appareil buccal des Simuliides. C.R. Soc. Biol. (Paris), $95: 1336-38$.

(1927). - L'appareil buccal de Corizoneura schwetzi et C. inornata. Rev. Zool. Afr., 15 : 125-142.

(1928). - Sur les tubérosités mandibulaires des Simuliides. C.R. Soc. Biol. (Paris), $99: 910-11$.

Patton (W. S.) et Cragg (F. W.) (1913). - A textbook of medical entomolog!, Christ. Lit. Soc. for India, 767 pp.

- et Evass (A. M.) (1929). - Insects, Ticks, Mites and venomous animals of medical and veterinary importance. Part I, Medical, $786 \mathrm{pp}$. 
P'rixgle (J. W. S.) (1948), - The gyroscopic mechanism of the halteres of Diptera. Phil. Trans., (B), $233: 347-384$.

Rubtzov (I. A.) (1956). - Simuliida. Faune de I'U.R.S.S., 6, (6), Moscou, 859 pp. Ś́GuY (E.) (1951). - Diptères in P. P. Grassé : Traité de Zoologie, 9, (1) : 448744, Messon édit., Paris.

(1940). - Faune de France, 36, Diptèies Nématocères (Fungivorida, Lycoriida....., Blepharoceridæ). P. Leehevalier édit., Paris.

(1926). - Faune de France, 13, Diptères Brachyec̀res, P. Lechevalier édit., Paris.

SMART (J.) (1935). - The internal anatomy of the Black-fly Simulium ornatum Mg. Ann. Trop. Med. and Parasitolog., $29: 161-170$.

SMrth (J. B.) (1890). - A contribution towards the knowiedge of the mouth parts of the Diptera, Trans. Amer. Ent. Soc., $17: 319$.

SNodgrass (R. E.) (1944). - The feeding apparatus of biting and sucking insects affecting man and mammals. Smithsonian Misc. coll., vol. 10\%, n" 7, $113 \mathrm{pp}$.

TÉTRY (A.) (1948). - Les outils chez les ètres vivants, coll. L'Avenir de la Seience, Gallimard édit., Paris.

Vimaen (A.) (1924). - Structure des pièces buccales des Blepharoceridix européens. Ceskoslov. ent. spol. Jubilejni Sbornik, Prague, pp. 136-143 (en tchèque).

WAsson (M.) (1950). - Contribution à l'étude de i'onchocercose humaine africaine. Soc. Belg. Méd. Trop., 30, (4) : 667-863.

\section{ANALYSE}

Satyn Yamaguti. - Systema helminthum. Vol. I. The Digenetic Trematodes of Vertebrates. Interscience publishers, New-York \& London. 1958. - Part I : xI + 979 p., Portrait frontispice. Part II : p. 980-1575, pl. I-CVI, fig. 1-1302. 90 dollars (48.750 fr. les deux parties).

Cet ouvrage est l'aboutissement d'un travail commencé il y a une trentaine d'années ; il est riche de substance. La partie I comprend un avantpropos (par E. W. Price), une préface, une introduction, une définition des Digénétiques (caractéristiques de la morphologie externe et interne), puis un compendium commenté de l'ensemble des Digénétiques, où sont considérés successivement ceux des Poissons, ceux des Batraciens, ceux des Reptiles, ceux des Oiseaux, ceux des Mammifères. On appréciera lä commodité de cette division, bien qu'elie ait l'inconvéniènt de disperser les genres et espèces d'une même famille, quand cette famille a des représentants chez des hôtes appartenant à plusieurs ordres. Pour les Digenea de Poissons, Yamaguti a admis 54 familles, dont une seule pour ies Gasterostomata, et 53 (dont 4 nouvelles) pour les Prosostomata ; pour 